\section{NEWS SECTION}

\section{Composers}

HENK BADINGS. Sonata for flute and harp (UK première)-6 February / Purcell Room / David Davies, Mary Ann Sherman.

ERIK BERGMAN. Borealis for 2 pianos and percussion (première)-I9 November $1983 /$ American University, Washington / Washington Music Ensemble. Violin Concerto (première)-I I May / Mainz / Hannele Segerstam, Staatsphilharmonie Rheinland-Pfalz.

PIERRE BOULEZ. Notations $I I$ (première)-7 March / Paris / Orchestre de Paris c. Daniel Barenboim.

JUSTIN CONNOLLY. Tesserae $B$ for flute and piano (première)-I I January / Purcell Room / Richard Davis, Simon Parkin.

EDWARD COWIE. Harp Concerto (première)-I5 January / Newcastle / Frances Kelly (harp), Northern Sinfonia c. Richard Hickox. American Symphony (première)-I February / Liverpool / Royal Liverpool PO c. Marek Janowski.

MARTIN DALBY. Scotch Rhapsody for viola and piano (première)-II January / Purcell Room / Sally Beamish, Simon Parkin.

FREDERICK DELIUS (d. 1934). Suite for violin and orchestra (première)-28 February / BBC broadcast.

MORTON FELDMAN. Violin and orchestra (première)-12 April / Frankfurt / Paul Zukovsky (vln), Frankfurt Radio SO c. Christóbal Halffter.

ERICA FOX. Kaleidoscope (première)-30 January / Wigmore Hall / Endymion Ensemble c. Howard Williams. Erica Fox was awarded the 1983 Gerald Finzi Composition Award for this work.

ROBERT GERHARD (d. 1970). Symphony No. 3 'Collages' (Dutch première)-12 April / The Hague / Residentie Orchestra c. Hans Zender.

PHILIP GLASS. Echnaton, opera in 3 acts (première)'-24 March / Stuttgart Staatsoper / c. Dennis Russell Davies.

HK GRUBER. Charivari (Austrian première)-22 January /Vienna / Austrian Radio SO c. Ivan Fischer.
PETER MICHAEL HAMEL. Von Traum und Tod, 3 orchestral pieces from the music-theatre work Ein Menschentraum (première)-26 March / Gelsenkirchen / State Orchestra c. Uwe Mund.

JONATHAN HARVEY. Easter Orisons (première) - I5 January / Newcastle / Northern Sinfonia c. Richard Hickox. Four Images after Yeats: Purgatory (première)-12 February / Wigmore Hall / Philip Martin (piano).

KLAUS HUBER. String Quartet No. 2 (première)-8 April / Munich / Berne String Quartet.

MINNA KEAL. Symphony No. I (première)-I3 February / St. John's, Smith Square, London / National Centre for Orchestral Studies c. Adrian Leaper.

VOLKER DAVID KIRCHNER. Passion for bass solo, speaker, children's choir, mixed chorus, and orchestra (staged première)-30 December 1983 / Wiesbaden / Hessisches Staatstheater / Eduard Wollitz (bass), choreography by Roberto Trinchero c. Siegfried Kohler.

BENJAMIN LEES. Fantasy Variations for piano (première)-I February / New York / Emanuel Ax.

KENNETH LEIGHTON. Dance Suite No. 3 (première)-25 February / Queen's Hall, Edinburgh / Edinburgh Symphony Orchestra c. Kenneth Leighton.

GYÖRGY LIGETI has been awarded the 1984 Prix Maurice Ravel.

JONATHAN LLOYD. Wind Quintet (première)-5 January / Purcell Room / Varèse Ensemble.

RICCARDO MALIPIERO. Nottumo for cello and orchestra (première)-29 January / Milan festival 'Musica nel nostro tempo' / c. G. Bernasconi.

IGOR MARKEVITCH (d. 1983). Le Paradis Perdu (Austrian première)-23 March / Vienna / Austrian Radio SO and Chorus c. Gerd Albrecht.

DAVID MATTHEWS. Serenade (UK première)-I3 April / Queen Elizabeth Hall / English Chamber Orchestra c. Raymond Leppard.

PETER PAUL NASH. Inventions for orchestra (première)-13 February / St. John's, Smith Square / National Centre for Orchestral Studies c. Adrian Leaper. 
KRZYSTOF PENDERECKI. Lux Aetema for solo, chorus and orchestra (première)-23 November 1983 / Washington / National SO and Chorus c. Mstislav Rostropovich. Penderecki was awarded the Sibelius Prize in Helsinki in October I983.

STEVE REICH. The Desert Music for chorus and orchestra (première)-I 7 March / Cologne / Cologne Radio SO and WDR Chorus c. Peter Eötvös.

WOLFGANG RIHM. String Quartet No. 5 (première)-9 December 1983 / Brussels Festival / Arditti Quartet. Double Concerto for viola, cello, and small orchestra (première)-I 8 January / Baden-Baden / Sudwestfunk Orchestra.

ROBERT SAXTON. Piccola Musica per Luigi Dallapiccola (UK première)-I I December I983/ Purcell Room / Koenig Ensemble.

ALFRED SCHNITTKE. Music for piano and orchestra (UK première)-I February / Manchester (BBC Studio 7 Concert) / Dmitri Alexeev, BBC Philharmonic c. Edward Downes.

WOLFGANG VON SCHWEINITZ. Adagio (première)-I I February / Berlin / Members of Berlin Radio SO.

KURT SCHWERTSIK. Skizzen und Entwüfe (US première)-2o February / San Francisco / Kronos Quartet.

ROBERT SIMPSON has completed his Tenth String Quartet; he has also rescored his The Four Temperaments, originally for brass band, for consort brass, and his Quintet for clarinet, bass clarinet and 3 double basses for clarinet, bass clarinet, violin, viola, and cello.

HOW ARD SK EMPTON. The Gipsy Wife's Song (première)-27 February / Wigmore Hall / Suoraan.

ROGER SMALLEY. String Quartet (UK première)—27 March / Purcell Room / Arditti Quartet.

RONALD STEVENSON. Fantasia Polifonica for harp (première)-8 February / Wigmore Hall / Satu Salo.

KARLHEINZ STOCKHAUSEN. Protest for trumpet; Zugenspitzentanz for piccolo (premières)-19 February / Illinois University / Kathinka Pasveer, Markus Stockhausen.
GILES SWAYNE. Symphony (première)-I June / Queen Elizabeth Hall / English Chamber Orchestra c. Stephen Barlow.

GERMAINE TAILLEFERRE, last survivor of 'Les Six', died in Paris on 7 November 1983.

SIR MICHAEL TIPPETT. The Blue Guitar (première)-9 November 1983 / Pasadena / Julian Bream. Festival Brass with Blues (première)-6 February / Hong Kong Festival / Fairey Band c. Howard Williams. The Mask of Time (première) - 5 April / Boston / Fay Robinson, Yvonne Minton, Robert Tear, Robert Cheek, Boston Symphony Orchestra and Chorus c. Sir Colin Davis. Sir Michael was awarded the Order of Merit on 20 December 1983 .

JUDITH WEIR. Sextet for piano and wind quintet (première)-I I February / Wigmore Hall / Nash Ensemble.

IANNIS XENAKIS. Pleiades for percussion (UK première)-8 February / London, Bloomsbury Theatre / Les Percussions de Strasbourg.

DOUGLAS YOUNG. Dreamlandscapes (premièrc)-2 February / Purcell Room / Peter Hill (piano). Chamber Music, 14 Joyce Songs (première)-22 February / Purcell Room / Margaret Ficld, Douglas Young.

\section{Periodicals}

\section{CONTACT}

No. 27, Autumn 1983

Christopher Fox, Walter Zimmermann's Local Experiments, pp. 4-9. Adrian Thomas, The Music of Henryck Mikolaj Górecki: the First Decade, pp. 10-20. David Jeffries, Tim Souster, pp. 20-27. Richard Barrett, Peter Wiegold, pp. 28-32. Graeme Smith, John Cage's 'Roaratorio': the Uses of Confusion, pp. 43-45.

\section{MUSICA}

6. November-December 1983

Carl Dahlhaus, Die Fuge als Präludium. Zur Interpretation von Schönbergs Genesis-Komposition Opus 44, pp. 522-524. Franz Zaunschirm, Cesar Bresgen: 'Requiem für Anton Weberm'. Cesar Bresgen zum 7o. Geburtstag, pp. 525-528.

I, January-February 1984

Manfred Trojahn, annotation zur improvisation, pp. 9-10. Walter Zimmermann, Nichts geht mehr, Die Improvisation und der Komponist, pp. I I-I3. 